\title{
Antiretroviral prescriptions with potential drug-drug interactions from general practitioners and specialists
}

\author{
Norah L Katende-Kyenda, M S Lubbe, J H P Serfontein, I Truter, J Bodenstein
}

To the Editor: It has been estimated that globally about 38.6 million people were infected with HIV by $2005,{ }^{1}$ with about 5 million of them living in South Africa (SA). The World Health Organization estimated that 4.7 million people living in sub-Saharan Africa urgently needed antiretroviral therapy (ART). In that year SA implemented prescribed minimum benefits (PMBs) for HIV/AIDS in the private health care sector. ${ }^{2}$ Despite the increased availability and affordability of ART in SA, only 60000 people were receiving ART through medical aid schemes by mid- 2005 . $^{3}$

Antiretrovirals (ARVs) have transformed HIV/AIDS into a chronic disorder that can be managed effectively. The right of all HIVinfected adults and children to receive standard care is endorsed by the SA HIV Clinicians Society (SAHIVCS), ${ }^{4}$ with ART guidelines recommending different combinations. The rapid approval of new drugs resulted in an increased risk of prescribing errors, dispensing of incorrect dosages/dose frequencies, and incorrect reporting of drugs by the patient to the prescribers, ${ }^{5}$ all leading to treatment failure.

Drug-drug interactions (DDIs) are an under-recognised consequence of medication prescription errors, resulting in significant health care costs. ${ }^{6}$ Since DDIs determine positive and negative consequences of treatment for HIV-infected patients, recommendations to avoid some drug combinations and to adjust dosages of some co-administered drugs were formulated by both the SAHIVCS ${ }^{4}$ and the Department of Health. ${ }^{7}$ The private health care environment recommends the Aid for AIDS ART guidelines, which are similar to those of the SAHIVCS (Table I).

We investigated the prevalence of prescriptions with potential DDIs between ARVs from general practitioners (GPs) and specialists (SPs) for patients in different age groups in the private health care sector, and evaluated the prescribed daily dosages (PDDs).

\section{Methods}

A non-experimental, retrospective, quantitative study was performed on data from a South African Pharmacy Benefit Management (PBM) company from 1 January 2005 to 31 December 2007. ARV drug names were classified according to the pharmacological groups in the Monthly Index of Medical Specialities (MIMS) and the Regulations to the Medicine and Related Substances Act (Act 101 of 1965). Prescribers of ARVs were divided into GPs and SPs.

\footnotetext{
Department of Pharmacology, Walter Sisulu University, Mthatha, E Cape Norah L Katende-Kyenda, PhD Pharmacy Practice

Medicine Usage in South Africa (MUSA), North-West University M S Lubbe, PhD Pharmacy Practice

J H P Serfontein, PhD Pharmacy Practice

Pharmacy Practice and Drug Utilisation Research, Nelson Mandela Metropolitan University, E Cape

I Truter, PhD Pharmacy Practice
}

Department of Pharmacology, University of KwaZulu-Natal, Durban J Bodenstein, PhD Pharmacology
Potential DDIs between ARVs were identified and classified as clinically major, moderate or minor. ${ }^{9}$ DDIs were assigned documentation levels of established, probable or suspected with significance ratings of $1,2,3,4$ and 5 . We focused only on DDIs with clinical significance rating of 2 , this rating being the most common between ARVs.

We evaluated potential DDIs between ARVs using PDDs. It is important that the PDD be related to the diagnosis made for the prescribed medication. The reference guides used to evaluate PDDs were according to the recommended ARV dosing. . $^{4,78}$

Basic descriptive statistics, i.e. frequencies, the arithmetical mean (average), standard deviations and effect sizes (Cohen's $d$ ) were used to characterise the study sample, and were calculated using the computer software Statistical Analysis System (SAS) for Windows 9.1. The age groups used were as follows: group $1-0 \leq 12$ years, group $2->12 \leq 19$ years, group $3->19 \leq 45$ years, group $4->45 \leq 59$ years, and group $5->59$ years.

Confidentiality of information was maintained, as no patient, medical practice, pharmacy or medical scheme could be identified. Permission to conduct the study was granted by the PBM company, and approval was obtained from the research and ethics committees of North-West University, Potchefstroom campus, (ethical number 07M01) and Walter Sisulu University, Mthatha campus.

\section{Results}

The study was performed on 49995,81096 and 88988 ARV prescriptions prescribed to 7664,10162 and $10061 \mathrm{HIV}$ patients in 2005, 2006 and 2007, respectively. ARV prescriptions represented $0.59 \%$ ( $N=49995), 0.90 \%(N=81096)$; and $1.11 \%(N=88988)$ of the claims for the 3 years.

In the 3 years, GPs prescribed more ARV prescriptions than SPs, and these increased from 2005 to 2007. The highest proportion of prescriptions was for three ARV items, followed by two ARV items. No practically significant differences were found between the average numbers of ARV medicine items per prescription $(d<0.8)$ claimed per year for the different years.

ARV prescriptions from GPs with potential DDIs and incorrect PDDs increased from $12.33 \%$ in 2005 to $24.26 \%$ in 2007 ; those from SPs increased from $15.46 \%$ in 2005 to $35.30 \%$ in 2006 and decreased to $33.16 \%$ in 2007 . The highest numbers of incorrect PDDs with DDIs were identified in ARV combinations of lopinavir/ritonavir $1066.4 \mathrm{mg} / 264 \mathrm{mg}$ with efavirenz $600 \mathrm{mg}$, and lopinavir/ritonavir $1066.4 \mathrm{mg} / 264 \mathrm{mg}$ with nevirapine $400 \mathrm{mg}$, followed by indinavir $1600 \mathrm{mg}$ with ritonavir $800 \mathrm{mg}$, ritonavir $600 \mathrm{mg}$ with efavirenz 600 $\mathrm{mg}$, and saquinavir $800 \mathrm{mg}$ with efavirenz $800 \mathrm{mg}$, for both GPs and SPs. The highest number of prescriptions were prescribed by GPs to patients in the age group $>19 \leq 45$ years, followed by the age group $>45 \leq 59$ years.

\section{Discussion}

We aimed to determine the prevalence of ARV prescriptions with potential DDIs and to evaluate their PDDs with specific reference to prescriber and age group. DDIs are of particular concern in HIV patients receiving highly active antiretroviral therapy. Although combination ARV treatment is potent and effective for HIV infection, ARVs frequently interact among themselves as many are metabolised 
Table I. Recommended drug combinations in the private sector ${ }^{8}$

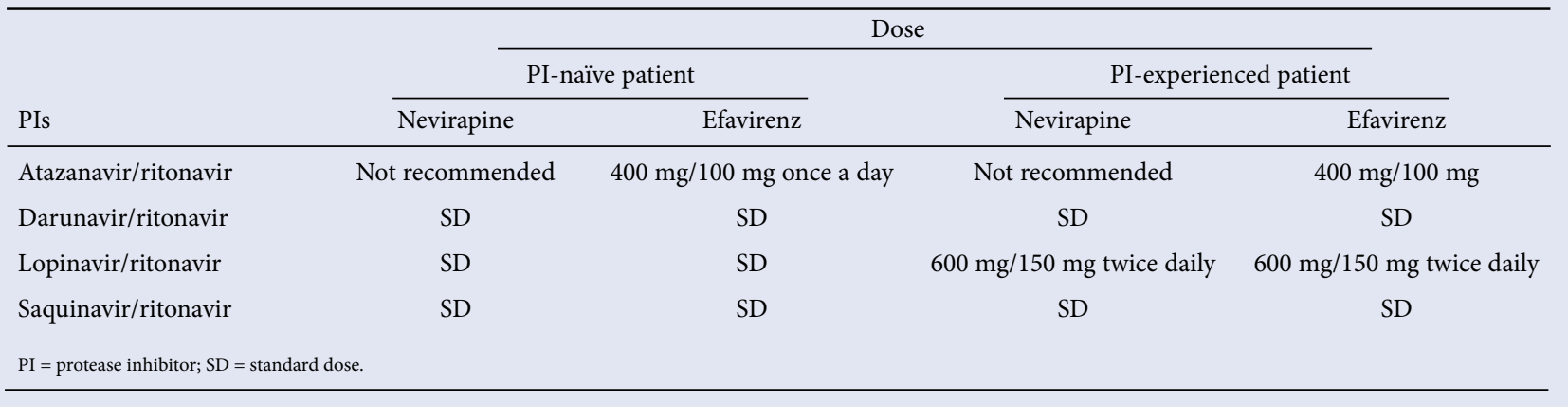

through the same CYP450 system. ${ }^{10}$ These interactions support the recommendations to avoid some combinations of drugs and to adjust the dosage of other drugs when co-administered.

The ARV regimens most identified with incorrect PDDs were between lopinavir/ritonavir (PI) prescribed at PDDs $1066.4 \mathrm{mg} / 264$ $\mathrm{mg}, 4500 \mathrm{mg} / 3999 \mathrm{mg}$ and $1599.6 \mathrm{mg} / 264 \mathrm{mg}$ with efavirenz (non-nucleoside reverse transcriptase inhibitor) (NNRTI), then the same with nevirapine. These were followed by indinavir (PI) with ritonavir (PI), saquinavir (PI) with efavirenz, and saquinavir with ritonavir. All these combinations were prescribed to patients aged $>19 \leq 45$ years. The standard adult dose for lopinavir/ritonavir is $400 \mathrm{mg} / 100 \mathrm{mg}$ twice daily or $800 \mathrm{mg} / 200 \mathrm{mg}$ once daily. ${ }^{7} \mathrm{~A}$ limitation of this study was that information about whether patients were HIV naïve or experienced was not available. Once-daily dosing for lopinavir/ritonavir is only recommended for treatmentnaïve patients, and not for patients receiving efavirenz, nevirapine or nelfinavir. When lopinavir/ritonavir is given with efavirenz or nevirapine, the recommended dose for treatment-experienced patients is $600 \mathrm{mg} / 150 \mathrm{mg}$ twice daily with food. ${ }^{7,8}$

Furthermore, the results revealed that GPs prescribed lopinavir/ ritonavir for patients in the age group $0 \leq 12$ years. Another limitation of this study was that the weights for children were not available and it was not clear to which weight and age categories of patients this co-formulation was prescribed. The safety, efficacy and pharmacokinetic profiles of lopinavir/ritonavir in children under the age of 6 months have not been established. The paediatric dose for lopinavir/ritonavir as recommended in the SA ART guidelines is 12 $\mathrm{mg}$ lopinavir/kg twice daily for children weighing $<15 \mathrm{~kg}$ and $10 \mathrm{mg}$ lopinavir/kg for children $\geq 15 \mathrm{~kg}$. In this case lopinavir/ritonavir was prescribed in a higher PDD, considering one capsule of lopinavir/ ritonavir to be $133.3 \mathrm{mg} / 33.3 \mathrm{mg}$, with the maximum dose not exceeding $399.9 \mathrm{mg} / 99.9 \mathrm{mg}{ }^{7}$ If given in higher PDDs the patient may therefore experience side-effects such as diarrhoea, nausea, vomiting, hyperlipidaemia and glucose intolerance. ${ }^{7}$

We observed that ritonavir was given with other PIs in PDDs of $3000 \mathrm{mg}, 1200 \mathrm{mg}, 800 \mathrm{mg}$ and $600 \mathrm{mg}$. The recommended dosages of $100 \mathrm{mg}$ capsules or $600 \mathrm{mg} / 7.5 \mathrm{ml}$ solutions in adults are $600 \mathrm{mg}$ every 12 hours (when ritonavir is used as sole PI). As a pharmacokinetic booster for other PIs, the recommended dosage is 100 - $400 \mathrm{mg}$ per day in two divided doses. ${ }^{7}$ Boosted PI regimens that utilise a low dose of ritonavir (100 - $200 \mathrm{mg}$ ) appear to offer the best balance of efficacy and tolerability.

\section{Limitations}

Limitations of this study include non-availability of patient clinical data to do in-depth clinical evaluation of the potential DDIs and
PDDs. The extent to which potential DDIs actually occurred could therefore not be determined. Information on HIV-naïve or experienced patients, CD4 values and viral loads were not available from the database. The clinical relevance of the identified potential DDIs was evaluated according to criteria in the literature. Information about the pharmacists who dispensed the prescriptions was not available. Pharmacists in collaboration with the prescriber could have detected and corrected prescriptions with potential DDIs and incorrect doses, this being one of the primary responsibilities of specific pharmacists. However, the results emphasised the possibility of DDIs that could have caused severe problems. A further limitation of the study was that no differentiation could be made between the prescription patterns of dispensing doctors versus pharmacydispensed prescriptions.

\section{Conclusion}

Our results emphasise the important role of prescribers and pharmacists in ensuring the appropriate use of ARVs. The importance of educating prescribers and dispensers by medical schemes or PBM companies in the private health care sector is also emphasised.

We gratefully acknowledge the managers of PBM who provided the data for the research project.

\section{References}

1. UNAIDS/WHO. AIDS epidemic update. Global Summary. December 2007. Geneva, Switzerland. www. uunaids.org (accessed 14 June 2008).

2. Erasmus S. Medical schemes in South Africa. HIV becomes prescribed minimum benefit. http://www. health24.com/medical/condition_centres/777-792-2002-2007,30583.asp (accessed 19 July 2007).

3. Johnson L. Estimated numbers of patients on antiretroviral treatment in the South African private Johnson L. Estimated numbers of patients on antiretroviral treatment in the South African private documents_v2/aids_ana/AAAO_Jan_Feb2006.pdf (accessed 29 March 2011).

4. South African HIV Clinicians Society Clinical Guidelines. Antiretroviral therapy in adults. http://www. hst.org.za/uploads/files/clincalguide_adults.pdf (accessed 25 January 2009).

5. Arshad S, Rothberg M, Rastegar DA, et al. Survey of physician knowledge regarding antiretroviral medications in hospitalized HIV-infected patients. Journal of the International AIDS Society 2009;12:1.

6. Heelon M, Skiest D, Tereso G, et al. Effect of a clinical pharmacist's interventions on duration of antiretroviral-related errors in hospitalized patients. Am J Health Syst Pharm 2007;64:2064-2068.

National Department of Health South Africa. 2005. ARV drug choices for children. In: Guidelines for the Management of HIV-infected Children. Jacana Media: Pinetown Printers.

8. Aid for Aids. Selecting Drug Combinations. AfA Clinical Guidelines. 2005. http://aidforaids.co.za/ ex_MEDSCHEME_VS07/Documents/AFA/Guidelines_book_Final.pdf (accessed 25 May 2008).

9. Tatro DS. Drug Interaction Facts. St Louis: Facts and Comparisons, 2005: 1-1699.

10. De Maat MM, Ekhart GC, Huitema AD, et al. Drug interactions between antiretroviral drugs and comedicated agents. Clin Pharmacokinet 2003;42:223-282. 